\title{
Descripción de las publicaciones de la Revista Otorrinolaringología y Cirugía de Cabeza y Cuello durante los años 2003 a 2008
}

\author{
Description of articles published in the journal \\ Revista Otorrinolaringología y Cirugía de Cabeza y Cuello from 2003 to 2008
}

Pilar Gajardo 01, Mario Cabezón D², Gabriel Rada G³.

\begin{abstract}
RESUMEN
Introducción: La literatura médica ha aumentado exponencialmente en los últimos años, y la otorrinolaringología no ha estado ajena a este fenómeno. Sin embargo estudios internacionales revelan que la mayoría de los artículos publicados en revistas de esta especialidad corresponden a diseños de estudio con un alto riesgo de sesgo.

Objetivo: Evaluar las publicaciones de la Revista Otorrinolaringología y Cirugía de Cabeza y Cuello, con respecto al diseño de los estudios incluidos, tema de la especialidad a la cual se refieren y lugar donde se realizaron los estudios.

Material y método: Se realizó un estudio retrospectivo donde dos revisores analizaron de manera independiente todos los artículos publicados en esta revista durante los años 2003 a 2008.

Resultados: El diseño de serie de casos fue el más utilizado, con 62,9\%. El 31,4\% de los trabajos abordó un tema de otología/otoneurología y 78,1\% de los artículos fueron realizados en un centro formador de especialistas en otorrinolaringología.

Discusión: Los artículos publicados en la revista seleccionada corresponden principalmente a estudios con alto riesgo de sesgo. En la actualidad existe un desbalance importante entre los temas que están siendo estudiados, y también entre los centros donde se está llevando a cabo la investigación en otorrinolaringología.
\end{abstract}

Palabras clave: Diseños de estudios, sesgo, publicaciones en otorrinolaringología.

\begin{abstract}
Introduction: Medical literature has experienced an important growth in past years, and otorhinolaryngology has not been the exception. However, international studies have revealed that most articles published in journals of this specialty correspond to study designs with a high risk of bias.
\end{abstract}

\footnotetext{
1 Médico de la Pontificia Universidad Católica de Chile. Tesista de Magíster en Ciencias Médicas, Mención Inmunología, Universidad de Chile.

2 Residente del Programa de Radiología de la Pontificia Universidad Católica de Chile.

3 Médico del Departamento de Medicina Interna, de la Unidad Docente Asociada, Hospital Sótero del Río y de la Unidad de Medicina Basada en Evidencia, Pontificia Universidad Católica de Chile.
}

Fuente de financiamiento: Propio 
Aim: To evaluate all articles published in the journal of Revista Otorrinolaringología y Cirugía de Cabeza y Cuello, according to the study design, area of the specialty that is involved and place where the studies were conducted.

Material and method: Two reviewers independently analyzed the original articles published in this journal from 2003 to 2008.

Results: Case series design was the most commonly used, with $62.9 \%$ of the studies. $31.4 \%$ of the articles were about otology/neurotology and $78.1 \%$ were performed in an educational and academic center where otorhinolaryngology specialists are trained.

Discussion: Articles published in the selected journal corresponded mainly to studies with a high risk of bias. There is an imbalance in the areas of the specialty that have been studied, and also in the places where these studies have been conducted.

Key words: Study design, bias, otorhinolaryngology journal.

\section{INTRODUCCIÓN}

En los últimos 20 años la literatura médica ha experimentado un crecimiento exponencial, encontrándose actualmente los profesionales de la salud con una inmensa cantidad de información disponible. A modo de ejemplo, la base de datos más importante de publicaciones biomédicas MEDLINE, indexa anualmente 700.000 nuevos artículos ${ }^{1}$. El campo de la Otorrinolaringología no ha estado ajeno a esta creciente avalancha de información. En 10 años hubo un aumento del total de publicaciones en las cuatro revistas otorrinolaringológicas de mayor circulación en Norteamérica desde 689 artículos en el año 1993 a 981 artículos en el año $2003^{2}$.

La integración de la investigación clínica a la toma de decisiones para los pacientes es lo que se conoce como Medicina Basada en la Evidencia (MBE). Esta herramienta consiste en "el uso explí- cito, racional y juicioso de la mejor evidencia disponible, en la toma de decisiones clínicas, incorporando las preferencias de los pacientes"3. Pero, ¿cómo saber cuál es la mejor evidencia disponible? Para responder esta pregunta es que se han jerarquizado los diferentes tipos de evidencia disponibles, según el riesgo de sesgo presente en cada diseño experimental (Tabla 1). En el caso de las decisiones sobre terapia, el nivel más alto de la jerarquía está constituido por revisiones sistemáticas de estudios clínicos randomizados (ECR) 0 ECR individuales.

Como se explicita en la definición de MBE, la toma de decisiones debe estar basada en la mejor evidencia disponible. Esto no significa que sólo podamos tomar decisiones cuando exista evidencia proveniente de los primeros niveles de la jerarquía, sino que a medida que utilizamos evidencia con mayor riesgo de sesgo, nuestras decisiones se basarán en supuestos menos certeros.

Tabla 1. Descripción de los niveles de evidencia para la toma de decisiones sobre terapia

\begin{tabular}{|clc|}
\hline Nivel de evidencia & Tipo de estudio & Riesgo de sesgo \\
\hline 1 & Estudios clínicos randomizados (ECR) & \\
& Revisiones sistemáticas y/o metaanálisis de ECR & + \\
3 & Estudios de cohorte & ++ \\
4 & Estudios de caso y control & +++ \\
5 & Estudios de serie de casos & ++++ \\
\hline
\end{tabular}

${ }^{\star}$ Adaptado de Oxford Centre for Evidence-based Medicine Levels of Evidence ${ }^{6}$. 
Según el estudio Bentsianov y cols. en otorrinolaringología en el año 1999, el 80\% de las recomendaciones terapéuticas se basaron en series de casos descriptivas, diseño correspondiente a un bajo nivel de evidencia y solamente el $7 \%$ en ECR ${ }^{4}$. Lo anterior fue confirmado por Yao y cols., quienes describieron que durante los años 2000 a 2005, solamente el $5 \%$ de los artículos de investigación publicados en las cuatro revistas otorrinolaringológicas de mayor circulación en Norteamérica correspondieron a ECR ${ }^{5}$. En la actualidad no existe información acerca del tipo de estudios clínicos publicados en la Revista Otorrinolaringología y Cirugía de Cabeza y Cuello.

\section{OBJETIVO}

Nuestro objetivo fue evaluar los estudios publicados en la Revista Otorrinolaringología y Cirugía de Cabeza y Cuello enfocándonos en el tipo de estudios publicados y su nivel de evidencia, en los temas médicos en ellos abordados y en la distribución según centro médico donde fueron realizados.

\section{MATERIAL Y MÉTODO}

En el contexto del proyecto de la Colaboración Cochrane de identificación de estudios clínicos randomizados a través de la revisión manual de revistas, la Unidad de Medicina Basada en Evidencia de la Pontificia Universidad Católica de Chile realiza una revisión de todas las revistas biomédicas chilenas. El presente reporte constituye un estudio observacional retrospectivo en el cual se revisaron manualmente todos los artículos de investigación publicados por la Revista Otorrinolaringología y Cirugía de Cabeza y Cuello entre los años 2003 y 2008. Si bien en algunos casos es difícil establecer un punto de corte acerca de qué se considera un estudio, incluimos todas aquellas publicaciones que responden a una pregunta de investigación, y en las cuales se describe una metodología o diseño experimental. Se excluyeron los artículos de revisión bibliográfica, editoriales, cartas al editor y reporte de casos clínicos.

Para la categorización según tipo de estudio cada artículo fue evaluado por dos revisores, quie- nes independientemente le asignaron alguna de las categorías descritas en la Tabla 1. En caso de haber discordancia entre los revisores ésta se resolvió de común acuerdo entre ellos.

La evaluación de los artículos según tema médico abordado fue hecha por un revisor, según la distribución temática de la Revista de la Sociedad Americana de Otorrinolaringología y Cirugía de Cabeza y Cuello (Otolaryngology-Head and Neck Surgery Journal).

La asignación del centro clínico se realizó en base a la filiación del primer autor del trabajo estudiado. En caso de haber discordancia entre la filiación del primer autor y el centro clínico donde se reclutaron los pacientes $y / 0$ se hicieron los análisis de los pacientes, este último criterio prevaleció.

Existen en la actualidad seis centros formadores reconocidos por la Sociedad Chilena de Otorrinolaringología y Cirugía de Cabeza y CueIlo, éstos son: Hospital Barros Luco Trudeau, Hospital Clínico de la Universidad de Chile, Hospital Clínico de la Pontificia Universidad Católica de Chile, Hospital del Salvador, Hospital Regional de Concepción y Hospital San Juan de Dios. Además de estos seis centros consideramos como centro formador al Instituto de Neurocirugía de Santiago y al Hospital Sótero del Río, ya que ambos reciben constantemente becados de la especialidad y forman una alianza docente con el Hospital del Salvador y el Hospital Clínico de la Pontificia Universidad Católica, respectivamente.

\section{RESULTADOS}

Se identificaron un total de ciento cinco estudios que cumplían con nuestro criterio de inclusión.

\section{Distribución por tipo de diseño de estudio}

El diseño de serie de casos correspondió ampliamente al modelo de estudio más usado con $62,9 \%$ del total y el diseño de estudio transversal correspondió al segundo más utilizado, con $17,1 \%$ de los artículos. De los ciento cinco estudios seleccionados para análisis, siete $(6,7 \%)$ correspondieron a estudios experimentales de laboratorio u otros diseños no aplicables a estudios observacionales, 
siendo clasificados dentro de la categoría de "otros diseños". La distribución de los artículos por tipo de diseño se muestra en la Figura 1.

Con respecto al diseño de estudio y el momento de la observación, la mayor parte de los estudios se realizaron en forma retrospectiva, con $55,2 \%$, seguido de los trabajos de corte transversal con $23,8 \%$. Los estudios prospectivos correspondieron a $14,3 \%$ de los estudios publicados en la revista.

Sólo $6,7 \%$ de los artículos publicados correspondieron a estudios que evaluaban pruebas diagnósticas, enfocándose la mayoría de los estudios en la descripción de características clínicas de casos. Nuestro trabajo no identificó ninguna revisión sistemática de la literatura.

\section{Distribución por tema}

Del total de artículos revisados $31,4 \%$ abordó una patología del área de otología/otoneurología, siendo éste el tema de publicación más frecuente en el período de tiempo estudiado. Luego le siguen en frecuencia los temas de otorrinolaringología pediátrica $(15,2 \%)$, otorrinolaringología general $(10,5 \%)$ y cirugía de cabeza y cuello (10,5\%). En la Figura 2 se observa la distribución de los artículos según tema abordado.

Al analizar los trabajos de otorrinolaringología pediátrica éstos presentan una distribución similar a los trabajos en adultos, ya que el $43,8 \%$ de ellos aborda una patología de otología/otoneurología, siendo este el tema más frecuente, seguido de patología adenoamigdalina $(37,5 \%)$, patología sinonasal $(12,5 \%)$ y finalmente cuerpo extraño en vía aérea $(6,3 \%)$.

Con respecto a la distribución de los artículos según país donde se realizó el estudio, solamente dos $(1,9 \%)$ de los trabajos fueron realizados en el extranjero, uno en Argentina y el otro en Brasil.

De los estudios realizados en nuestro país, veintiséis de los ciento cinco trabajos evaluados fueron realizados en el Hospital Clínico de la Universidad de Chile, siendo éste el centro con mayor número de

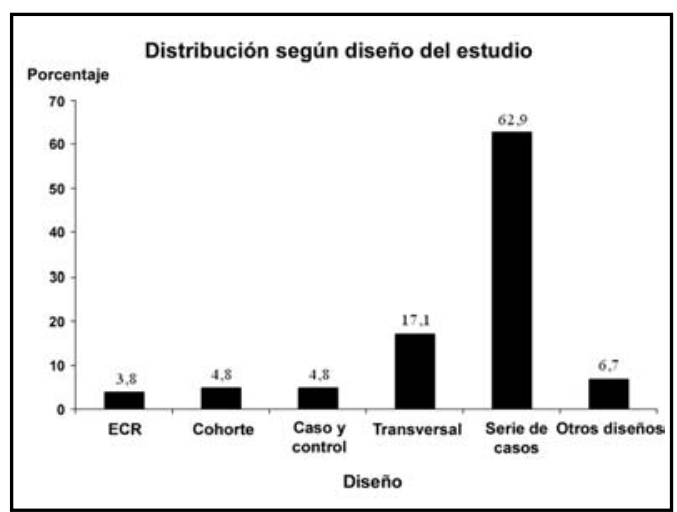

Figura 1. Distribución de los artículos publicados en la Revista de Otorrinolaringología y Cirugía de Cabeza y Cuello entre los años 2003 y 2008, según diseño de estudio utilizado.

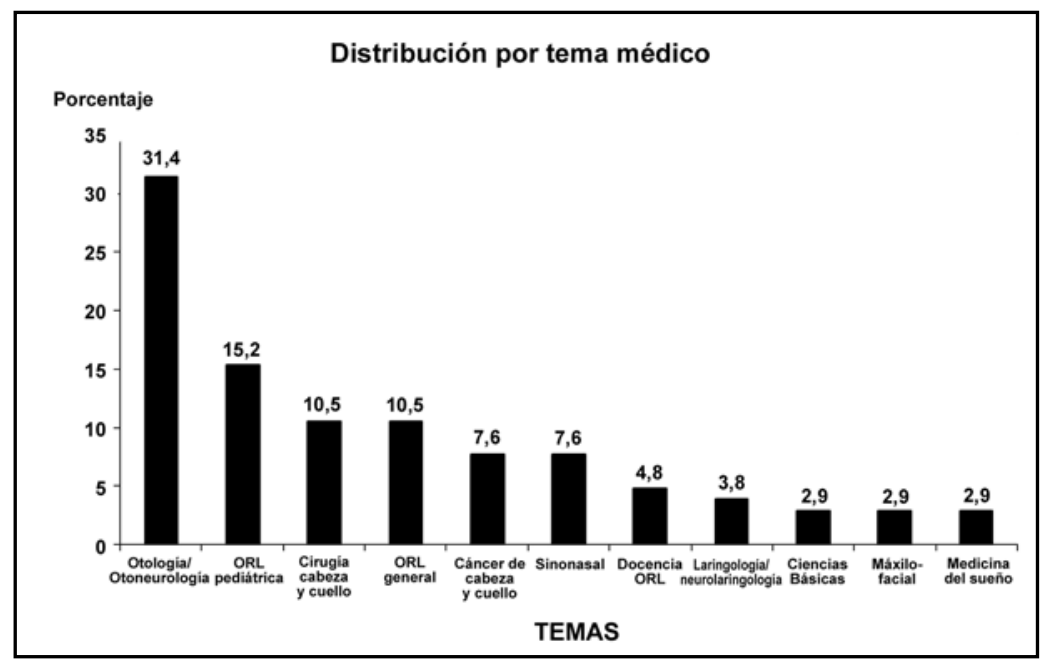

Figura 2. Distribución de los artículos publicados en la Revista de Otorrinolaringología y Cirugía de Cabeza y Cuello entre los años 2003 y 2008, según tema de la especialidad abordado. 
estudios publicados, seguido por el Hospital Clínico de la Pontificia Universidad Católica de Chile con veintiún artículos, abordando entre ambos el $45 \%$ del total de artículos publicados en el período de tiempo estudiado. En la Figura 3 puede apreciarse el aporte de cada centro al total de artículos publicados.

Al analizar los artículos según el centro, destaca que el $40,8 \%$ de ellos fueron realizados en Hospitales del Ministerio de Salud. De los realizados en centros no públicos, once (10,7\%) fueron realizados en clínicas o institutos médicos privados, siendo la Clínica Las Condes el centro privado con más publicaciones en este período, con cinco artículos.

Durante el período estudiado el $78,1 \%$ de los artículos publicados fueron realizados en centros formadores de especialistas en otorrinolaringología. El Hospital Clínico de la Universidad de Chile (HCUCH) y la alianza Hospital Clínico Pontificia Universidad Católica de Chile-Hospital Sótero del Río son los centros formadores con mayor número de artículos de investigación publicados en el período 20032008, con veintiséis y veinticinco artículos, respectivamente. La distribución de artículos según centros formadores puede observarse en la Figura 4.

\section{DISCUSIÓN}

Los autores de la Revista de Otorrinolaringología y Cirugía de Cabeza y Cuello durante los años 2003 a

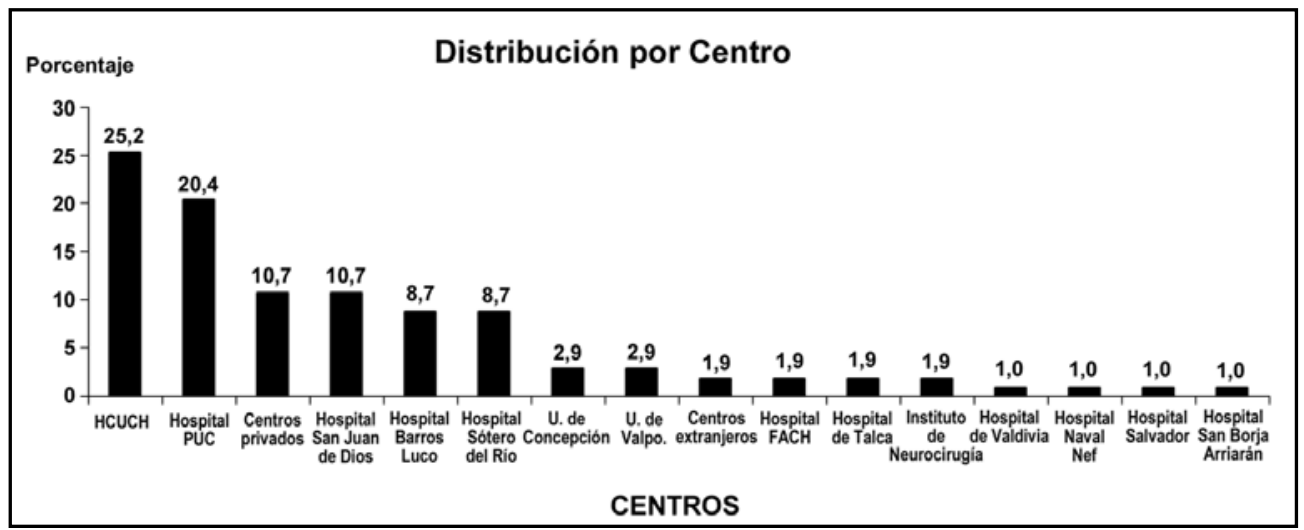

Figura 3. Distribución de los artículos publicados en la Revista de Otorrinolaringología y Cirugía de Cabeza y Cuello entre los años 2003 y 2008, según centro clínico donde se realizó el trabajo.

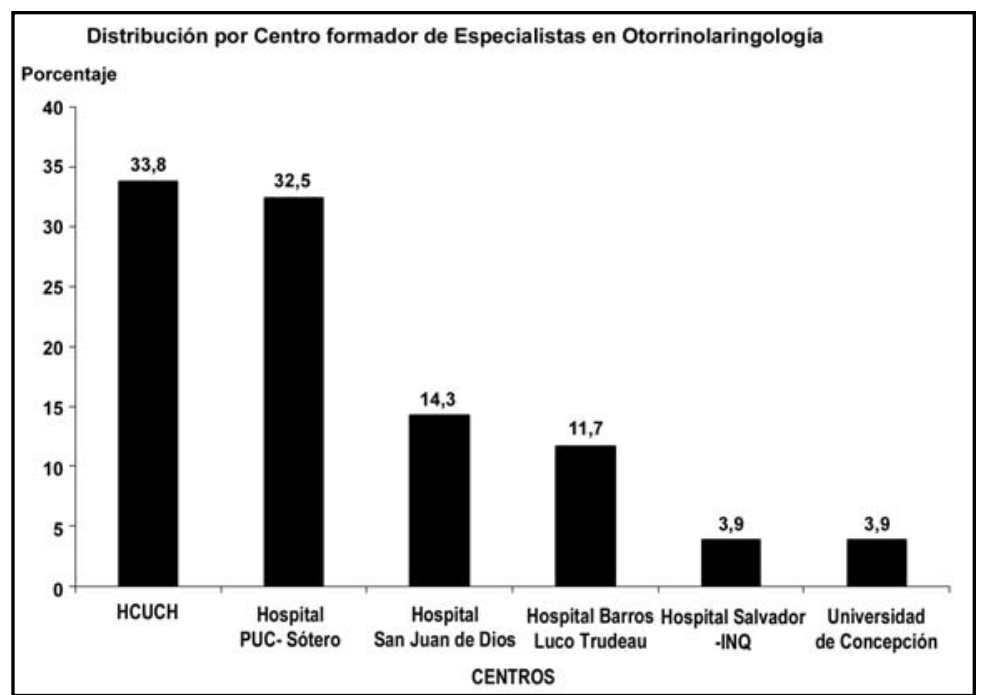

Figura 4. Distribución de los artículos publicados en la Revista de Otorrinolaringología y Cirugía de Cabeza y Cuello entre los años 2003 y 2008, realizados en centros formadores de médicos otorrinolaringólogos. 
2008 se inclinaron mayoritariamente por un diseño del tipo serie de casos. En general, este tipo de estudios describe la experiencia de un grupo de pacientes con un diagnóstico similar, permitiendo generar hipótesis, documentar nuevas enfermedades 0 efectos adversos de un determinado tratamiento. Sin embargo, difícilmente pueden sugerir 0 establecer causalidad, pues carecen de grupo de control. Es por eso que representan un nivel de evidencia con mayor riesgo de sesgo $0^{7}$.

Por otra parte, aquellos diseños considerados desde el punto de vista de la Medicina Basada en Evidencia, de mayor jerarquía, constituyen un menor porcentaje.

Con respecto a la distribución por tema, existe una marcada tendencia hacia la otología/ otoneurología, tanto en artículos de adultos, como pediátricos, lo cual creemos debe tomarse en cuenta, a modo de fomentar el desarrollo de nuevas áreas de interés, distintas a la otología, como las patologías sinonasales y laríngeas.

Es importante destacar que a partir del año 2007 se han incorporado en la revista artículos de ciencias básicas, que si bien en el período evaluado corresponden solamente al $2,9 \%$ de los artículos, indican el creciente interés de los médicos en ámbitos experimentales de laboratorio, como una herramienta complementaria al quehacer clínico.

Si bien es esperable que la mayoría de los artículos estudiados hayan sido realizados en centros formadores de especialistas en otorrinolaringología, creemos que existe un importante desbalance entre los centros, lo cual debe ser tomado como un signo de atención, para crear mayores instancias de investigación en los centros con menor aporte, ya que la investigación, tanto clínica como básica, corresponde a un pilar fundamental de la práctica docente y de la formación de futuros especialistas.

\section{CONCLUSIONES}

Las publicaciones de la Revista de Otorrinolaringología y Cirugía de Cabeza y Cuello durante los años
2003 a 2008, corresponden principalmente a estudios nacionales con diseños experimentales con alto riesgo de sesgo. Existe en este período una marcada tendencia a la publicación de artículos relacionados con la otología/otoneurología, lo cual pudiese reflejar que en nuestro país ésta es el área de la especialidad donde se están realizando mayores actividades de investigación, sin embargo, la mayoría son estudios observacionales.

Creemos que la información analizada en el presente trabajo puede servir a los especialistas en otorrinolaringología para ampliar las áreas de interés y fomentar la actividad de investigación básica y clínica de mejor calidad.

\section{BIBLIOGRAFÍA}

1. Pubmed. Disponible en: www.ncbi.nlm.nih.gov/pubmed/ [Consultado el 14 de Diciembre de 2009].

2. Wasserman JM, Wynn R, Bash tS, Rosenfeld RM. Levels of evidence in otolaryngology journals. Otolaryngol Head Neck Surg 2006; 134: 717-23.

3. Guyatt G, Rennie D. User's Guide to the Medical Literature: A Manual for Evidence-Based Clinical Practice. First ed; 2002.

4. Bentsianov BL, Boruk M, Rosenfeld RM. Evidence-based medicine in otolaryngology journals. Otolaryngol Head Neck Surg 2002; 126: 371-6.

5. Yao F, Singer M, Rosenfeld RM. Randomized controlled trials in otolaryngology journals. Otolaryngol Head Neck Surg 2007; 137: 539-44.

6. Howick J. Oxford Centre for Evidence-based Medicine Levels of Evidence. www.cebm.net; 2009.

7. Pita Fernández, S. Epidemiología. Conceptos básicos. En: Tratado de Epidemiología Clínica. Madrid; DuPont Pharma, S.A.; Unidad de epidemiología Clínica, Departamento de Medicina y Psiquiatría. Universidad de Alicante: 1995; 25-47.

Dirección: Dr. Gabriel Rada

Lira 63, Santiago

E mail: umbeuc@med.puc.cl 